\title{
Need for primary care providers to carry out annual oral cancer examinations
}

\begin{abstract}
Oral and/or oral pharyngeal cancer (OPC) has an approximately 53\% five-year survival rate in the United States. It is postulated that the rationale for this low survival rate is due to delayed diagnosis and treatment of OPC at its later stages. Recently the United States Preventive Services Task Force found insufficient evidence to recommend for or against oral cancer examination (OCE) by primary care providers (PCP), i.e., medical providers who are not dental providers or otolaryngologists. The purpose of the paper is to provide a logical discussion of the varied research on OCE, while presenting the evidence for annual opportunistic OCE in the primary care setting. Trained PCPs in OCE have the likelihood of detecting OPC at an early non symptomatic stage, which could result in lower treatment morbidity and mortality due to the disease. This review of the OCE research summarizes evidence that points to a logical benefit of OCE. Opportunistic annual non symptomatic OCE by PCPs who have been trained in OCE techniques may result in consistent early detection of OPC, particularly for patients at high risk for developing the disease.
\end{abstract}

Keywords: oral cancer examination, oral and oropharyngeal cancer, screening, primary care providers
Volume 2 Issue 2 - 2015

\author{
Alvin G Wee,' Lani M Zimmerman, ${ }^{2}$ Carol \\ H Pullen, ${ }^{2}$ Michael A Sitorius, ${ }^{3}$ Electra D \\ Paskett $^{4}$ \\ 'Department of Prosthodontics, Creighton University School of \\ Dentistry, USA \\ ${ }^{2}$ University of Nebraska Medical Center, USA \\ ${ }^{3}$ Department of Family Medicine, University of Nebraska \\ Medical Center, USA \\ ${ }^{4}$ Department of Internal Medicine, The Ohio State University, \\ USA
}

Correspondence: Alvin G Wee, Department of Prosthodontics, Creighton University School of Dentistry, Dental Department 160,VA Nebraska-Western lowa Health Care System, 4101 Woolworth Avenue, Omaha, NE 68105, USA, Tel (402) 290-6821, Email alvingwee@gmailo.com

Received: December 12, 2014 | Published: April 6, 2015

Abbreviations: OPC, oral pharyngeal cancer; OCE, oral cancer examination; PCP, primary care providers; PPV, positive predictive value; NPV, negative predictive value

\section{Introduction}

An estimated 42,380 men and women were diagnosed with oral and/or oropharyngeal cancer (OPC) in 2013, and of these, 7,890 deaths ${ }^{1}$ were reported. OPC is the sixth most common cancer in the United States for males. Higher rates of OPC are reported among minorities; OPC presently ranks as the fourth most common cancer among African American men. ${ }^{2}$ Nearly $90 \%$ of OPC occurs in patients older than 45years of age, with African Americans exhibiting a peak incidence nearly 10years earlier than other groups (usually diagnosed at 65-74 years of age). OPC also has one of the lowest five-year survival rates in the United States compared to any other cancer, at approximately a $53 \%$ survival rate in five years. ${ }^{3}$ The five-year survival rate for advanced cases is $19 \%$, compared to $78 \%$ for localized lesions. ${ }^{4}$ Surgical treatment for advanced-stage OPC is associated with high morbidity, including a decrease in quality of life. ${ }^{5,6}$ Poor overall survival rates of patients with OPC are due to treatment of the disease in its later stages, with only $33 \%$ of patients in the United States patients presenting at an early stage. ${ }^{3}$ Delayed diagnosis is proposed to be the most important determining factor behind this dire statistic. ${ }^{7,8}$ Recently the United States Preventive Services Task Force (USPSTF) found insufficient evidence to recommend for or against oral cancer examination (OCE) by primary care providers (PCP), i.e., medical providers who are not dental providers or otolaryngologists. ${ }^{9}$ The task force did not find adequate evidence that OCE and treatment of screen-detected OPC improved morbidity or mortality of this disease compared to OPC that was detected without screening. It further concluded that the current evidence was insufficient to assess the balance between benefits and harm when for PCPs perform OCE on non symptomatic adults.
The purpose of this paper is to provide a logical discussion of the varied research on OCE, while presenting the evidence for annual opportunistic OCE in the primary care setting. Trained PCPs in OCE have the likelihood of detecting OPC at an early non symptomatic stage, which could result in lower treatment morbidity and mortality due to the disease.

\section{Oral cancer examination strategies}

An important unusual characteristic of OPC relating to diagnosis is that it is non symptomatic at the premalignant and early stages. Patients become symptomatic when OPC is in the later stage. ${ }^{10}$ OCEs have the potential to greatly impact survival of patients with OPC, with few, if any, negative consequences to the patient. OCE essentially entails a visual inspection of the face, neck, lips, labial mucosa, and buccal mucosa, and gingiva, floor of the mouth, tongue, and palate. This is a simple, noninvasive, and painless examination that is acceptable to patients, cost effective, and potentially lifesaving. ${ }^{11}$ Secondary prevention of OPC consists of early detection by healthcare providers ${ }^{12,13}$ and interpretation of signs and symptoms of OPC by patients. ${ }^{14}$ Figure 1 show a modification of a conceptual framework that was proposed in $1998,{ }^{15}$ where the proposed OCE by PCP fits into the flow from diagnosis of OPC to cancer treatment. This figure elaborates how the diagnostic process takes place within the healthcare system and its influence on patient and professional delay. The American Cancer Society recommends routine OCE every three years for individuals 20years and older and annually for individuals 40years and older. ${ }^{16}$ Screening program approaches include population screening, targeted screening, or opportunistic screening. The objective is to reduce the treatment mortality and morbidity due to OPC, or to produce a cancer stage shift and improve the survival rate of patients with OPC across populations. 


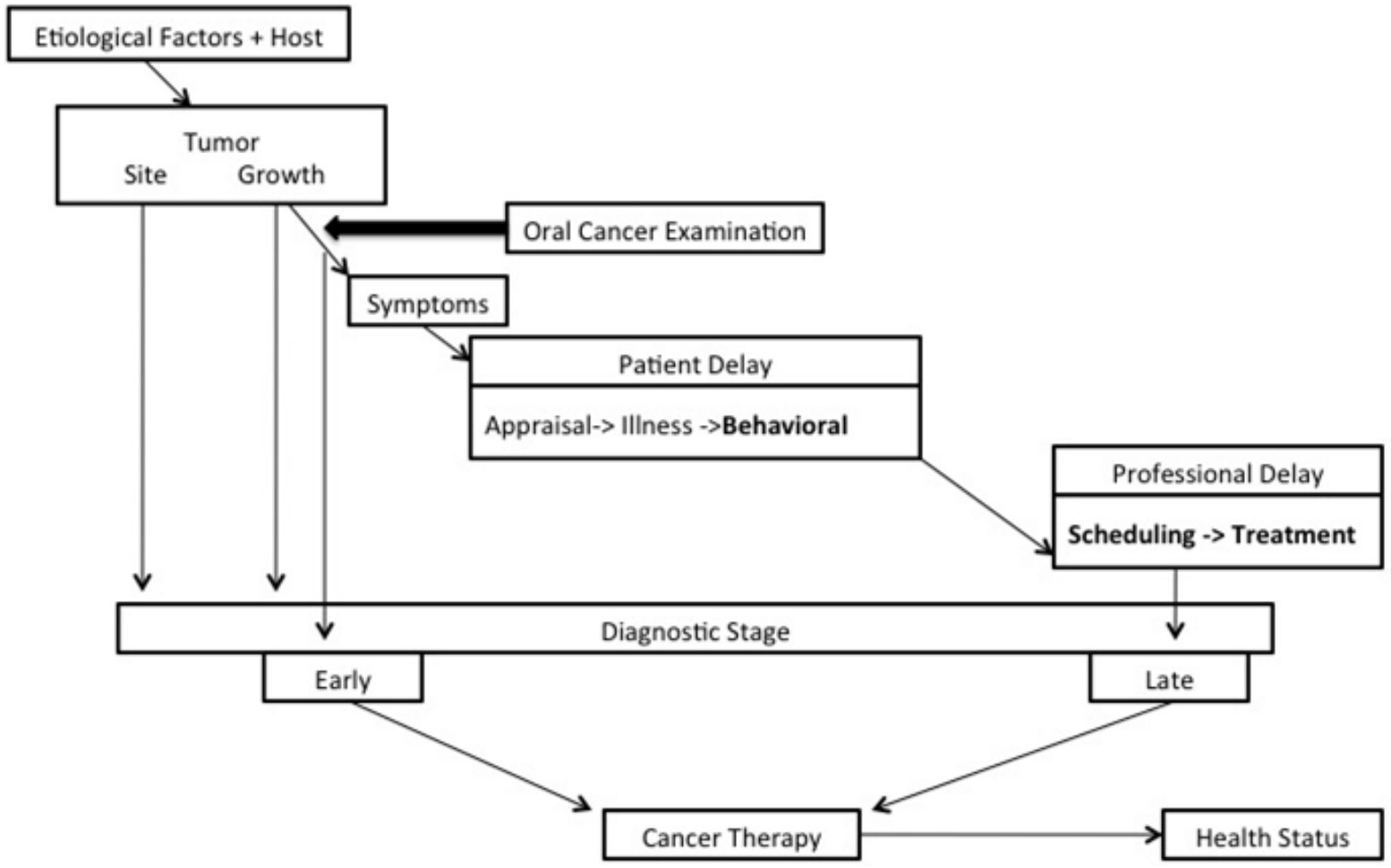

Figure I Conceptual framework for oral and/or oropharyngeal cancer diagnosis to treatment.

\section{Population screening}

Two recent reviews, one by the Cochrane Collaboration ${ }^{17}$ and the other by the American Dental Association Council on Scientific Affairs $^{18}$ both concluded that there is insufficient evidence to determine whether population screening carried out by OCE reduces the mortality for patients with OPC, especially for non symptomatic individuals seeking dental care. ${ }^{18}$ However, there is some evidence that OCE might help reduce death rates in patients who use tobacco and alcohol, while simultaneously producing a stage shift from latestage to early-stage diagnosis and improvement in survival rates across the population. ${ }^{17}$ Unfortunately, the evidence is limited to only one study that is associated with a high risk of bias. The study is a nine-year randomized controlled trial with 13 clusters and 191,873 subjects. ${ }^{19}$ The study on population screening of high-risk individuals who used tobacco or alcohol or both in the intervention cohort had a $43 \%$ reduction in mortality, compared to a $24 \%$ reduction in mortality for the control cohort. The finding was statistically significant. Unfortunately, population screening for OPC is cost-prohibitive because the prevalence of OPC is understood to be low in the United States. Comparatively, OPC prevalence is higher in India; it is the most common form of cancer in that country. It also accounts for the highest number of cancer-related deaths among men in India. ${ }^{20}$ A recent study showed that the cost-effectiveness of population screening for OPC in India (cost per life year) was $\$ 835$ (USD, U.S. Dollar) for all individuals eligible for OCE. For high-risk individuals, the cost per year was $\$ 156$ (USD). ${ }^{21}$ In summary, there is insufficient evidence that population screening reduces the mortality for patients with OPC. Thus, targeted screening is the next logical alternative.

\section{Targeted screening}

Targeted screening using OCE is more cost-effective for highrisk groups compared to screening the general population ${ }^{21}$ but there is a high noncompliance rate for subjects who are advised to get a biopsy. ${ }^{22}$ With the emergence of the human papillomavirus (HPV-16) as a contributory risk factor for OPC (especially tonsil, oropharynx and base of tongue cancer), nonsmokers and nondrinkers can also develop OPC. ${ }^{23,24}$ The prevalence of HPV in OPC globally was estimated to be $23.5 \%$ in $2005^{25}$ but in the United States, it is currently estimated to be around $80 \%$ to $95 \%{ }^{26}$ This increase in OPC from HPV is higher among Caucasians, particularly in the younger generation. ${ }^{27}$ This scenario has made it difficult for healthcare providers to target actual high-risk OPC groups for OCE without additional laboratory tests for patients' HPV status.

\section{Opportunistic screening}

The third strategy, providing opportunistic screening, is probably the most cost-effective and productive. ${ }^{28}$ When patients visit physicians, dentists, or any other healthcare provider (e.g., physician assistants or nurse practitioners) for routine care, an OCE can easily be accomplished as part of their healthcare visit. There is only one published study that reports on opportunistic screening for OPC. This longitudinal study (1983 to 1990) evaluated opportunistic OCE in Cuba by a stomatologist, as well as public oral cancer surveillance education. ${ }^{29}$ The study showed significant down staging of OPC diagnosis after the introduction of the educational program. The authors reported an increase from $22.8 \%$ to $48.2 \%$ of patients found to have stage one OPC and a decrease in stages two, three, and four from $77.2 \%$ to $51.8 \%$. 


\section{Percentage of individuals obtaining annual oral cancer examinations}

Despite the accessibility of structures and relative ease of completing OCEs, the 1992 National Health Interview Survey showed that only $14 \%$ of adults in the United States adults reported ever having had an OCE and only 7\% reported having had one during the past year. ${ }^{30}$ The 1998 National Health Interview Survey showed an increase, with $13.5 \%$ having had an OCE during the past year. $^{31,32}$ In 2008, 29.4\% of adults ages 18 years and older reported having ever had an OCE, which was a moderate increase from the national 1992 data. ${ }^{33}$ Alarmingly, the 2008 survey report contains a note that those most at risk for OPC (current smokers aged 40 years and older) were less likely to have had an OCE than former smokers or those who had never smoked. It is estimated that $71.2 \%$ of the United States population "visited the dentist or dental clinic within the past year for any reason", according to the 2008 Behavioral Risk Factor Surveillance System survey. ${ }^{34}$ Unfortunately, not all dentists in the United States perform OCE. The National Oral Cancer Survey of Dentists conducted in the United States in $1995^{35}$ showed that $81 \%$ reported conducting OCEs on all of their patients over 40years of age.

Furthermore, individuals who are less likely to visit a dentist are middle-aged and older edentulous, of a low socioeconomic status, or of African American or Hispanic ethnicity. These groups are similar to those at high risk for OPC. ${ }^{36-38}$ In the United States, individuals who are 65years and older and retired are usually covered by Medicare plans that do not provide dental coverage. ${ }^{39}$ Marginal and lowerincome groups also may not be able to afford to purchase private dental insurance and therefore, are not covered financially for dental examinations.

\section{Medical providers and nonsymptomatic oral cancer examination}

Individuals who have high-risk behaviors (e.g., smoking, drinking) for OPC are more likely to be covered by medical insurance than dental insurance. These individuals may have a higher tendency to have medical problems associated with poor health choices, such as smoking, alcohol consumption, or low vegetable intake. ${ }^{40}$ It is estimated that up to $75 \%$ of oral cancer cases may be attributed to tobacco and alcohol use. ${ }^{41}$ Other risk factors for OPC that could contribute to poor medical health include infection with Candida or bacterial flora and a compromised immune system. ${ }^{24}$ Individuals at high risk for OPC have a 4 to 6 times higher likelihood of seeing a non specialist PCP than a dentist. ${ }^{42,43}$ PCPs may reduce the delay in diagnosis of $\mathrm{OPC}^{44}$ by conducting routine non symptom-driven OCE in their practices for patients who are at high risk for OPC. Unfortunately, the frequency of self-report by physicians conducting routine OCE varies greatly, with less than 24\% in 2002 in Maryland ${ }^{45}$ to $49 \%$ of physicians in Massachusetts in $2009^{46}$. Surprisingly, in a survey of physicians at federally qualified health centers in Michigan in 2003 , more than $70 \%$ self-reported that they performed OCE. ${ }^{47}$ Findings from other studies that do not rely on physician self-reporting imply a lower frequency of routine OCE. In one study, more than $77 \%$ of 160 patients who were diagnosed with advanced OPC had received routine medical care in the preceding $3-24$ months. ${ }^{48}$ In another study, at least $94 \%$ of 130 patients diagnosed with head and neck cancer recalled visiting a medical provider during the preceding 24 months. ${ }^{49}$ Only $3 \%$ of internal medicine residents in an outpatient primary care clinic of an inner-city hospital provided OCE for their patients, as revealed in a review of 151 patient charts..$^{50}$
Physicians' lack of attempted or effective diagnosis from non symptomatic OCE may contribute to conclusion of the USPSTF regarding OCE. As cited previously, OPC in its earliest stages is often non symptomatic. It has been shown that medical providers who detect OPC during non symptomatic OCE consist primarily of dentists, hygienists, and oral maxillofacial surgeons..$^{51}$ Non symptom-driven OCE had a significantly lower clinical stage of diagnosis compared to symptom-directed OCE. ${ }^{51}$ Oral lesions detected by PCPs were usually found during a symptom-driven examination ${ }^{52}$. Furthermore, recent reports show that OPC patients who were not under the regular supervision and care of a dentist were more likely to have longer diagnostic delay. ${ }^{53}$ Such findings emphasize the need to provide education to PCPs on OPC risk factors, as well as OCE techniques. ${ }^{50}$ Regarding PCPs other than physicians, a survey of nurse practitioners showed that only a small percentage (19\% in Maryland ${ }^{54}$ and $39.3 \%$ in Florida ${ }^{55}$ ) felt their knowledge about OPC was current. Less than $50 \%$ of nurses in the United Kingdom perform OCE regularly ${ }^{56}$ while more than half of the nurse practitioners in Florida reported having never performed the procedure..$^{55}$ Some factors contributing to these statistics may include that nurse practitioners do not consider OCE to be part of their professional duty, while some may have inadequate training and/or uneasiness about examining the mouth. ${ }^{57}$

Only one small pilot study found in the literature presents information regarding the education of medical care professionals (three physicians, one physician assistant, and four registered nurses) on OPC. The education consisted of a one-day training divided into two sessions. The posttest training questionnaire indicated that their training was well received and would prompt them to increase their efforts to examine their patients for early signs of oral cancer and to counsel them about its risks. ${ }^{58}$

\section{Providing oral cancer examinations to populations not been screened}

There is a general consensus that PCPs would benefit from enhanced OCE skills to improve their performance in early detection of OPC.$^{59}$ Effective OCE by PCPs, particularly physicians, physician assistants, and nurse practitioners, is possible if the proper education is provided. ${ }^{12}$ PCPs are interested in obtaining continuing education on OPC and OCE. ${ }^{59}$ Nurse practitioners who completed continuing education on OPC in the past two to five years were approximately three times more likely to have a high score on knowledge of OPC compared to those who had never attended a course. ${ }^{54} \mathrm{~A}$ meta-analysis done on eight selected OCE studies shows that the weighted pool overall sensitivity is 0.85 and the specificity is 0.97 for diagnosing OPC. ${ }^{60}$ Educating patients to carry out self-OCE may not be efficacious, as shown in (Table 1), given low sensitivity and moderate positive predictive value. ${ }^{61,62}$ The sensitivity and positive predictive value of the OCE appears to increase as the education and training of the healthcare workers increase. The sensitivity and positive predictive value of the OCE increases from $59 \%$ to $94.3 \%$ and $31 \%$ to $86.6 \%$, respectively, when comparing OCE conducted by lay healthcare workers with a high school education and two to five days of OCE training ${ }^{63}$ versus OCE conducted by lay healthcare workers with university degrees and six weeks of OCE training. ${ }^{64}$ Because PCPs have higher education than the subjects in these studies ${ }^{63,64}$ it is certain that the sensitivity and positive predictive value for OCE by PCPs can be high. 
Table I Performance Characteristics of OCE

\begin{tabular}{|c|c|c|c|c|c|c|c|}
\hline Citation & Screened & Venue/Location & Gold Standard & Sensitivity & Specificity & PPV* & NPV* \\
\hline \multicolumn{8}{|c|}{ Patients' self OCE } \\
\hline Elango et al. ${ }^{62}$ & 34,766 & Subject's home/India & $\begin{array}{l}\text { Health Worker OCE >4 } \\
\text { weeks }\end{array}$ & $18 \%$ & $99.9 \%$ & $72 \%$ & $99 \%$ \\
\hline Scott et al. ${ }^{61}$ & 53 & Dental Office & Dentist performed OCE first & $33 \%$ & $54 \%$ & $17 \%$ & $73 \%$ \\
\hline \multicolumn{8}{|c|}{ OCE by Healthcare workers: high school education with 2 to 5 days training on OCE } \\
\hline Mehta et al. ${ }^{63}$ & 1,921 & $\begin{array}{l}\text { Subject's home/ } \\
\text { India }\end{array}$ & $\begin{array}{l}\text { Dentist carrying out } O C E>6 \\
\text { months }\end{array}$ & $59 \%$ & $98 \%$ & $31 \%$ & $99 \%$ \\
\hline \multicolumn{8}{|c|}{ Healthcare workers: university graduates with 6-week training on OCE } \\
\hline Mathew et al. ${ }^{64}$ & 2,069 & $\begin{array}{l}\text { Subject's home/ } \\
\text { India }\end{array}$ & $\begin{array}{l}\text { Physician carrying out OCE } \\
>6 \text { months }\end{array}$ & $94.3 \%$ & $98.3 \%$ & $86.6 \%$ & $99.3 \%$ \\
\hline
\end{tabular}

*PPV=Positive predictive value.

*NPV=Negative predictive value

\section{Future research direction}

Evidence from a variety of studies, then, shows less-thanconclusive OCE frequency by PCPs and suggests that training of PCPs in OCE may be needed to maximize its effectiveness. Such findings highlight the need for a randomized controlled trial in the United States of PCPs carrying out OCE, with long-term patient follow-up and analysis of treatment morbidity and mortality. To date, no such conclusive studies have been completed. Such scientific evidence will be necessary to allow the USPSTF to positively recommend that PCPs with training carry out routine annual non symptomatic OCE. Such a recommendation would result in PCPs beginning to consistently provide annual routine non symptomatic OCE to their patients and result in an eventual policy change. With the potential policy change, medical insurance companies would consider paying for OCE by PCPs.

\section{Conclusion}

Despite the long-standing poor five-year survival rates for patients with OPC in the United States, as well as the strong indicators that earlier diagnosis of OCP is critical to improve these rates, the USPSTF did not find strong evidence to recommend routine non symptomatic OCE by PCPs, given a paucity of data regarding OCE in general and a lack of a controlled randomized trials to establish an improvement in treatment morbidity and mortality as a result of OCE and early detection. However, this review of the OCE research summarizes evidence that points to a logical benefit of OCE. Opportunistic annual non symptomatic OCE by PCPs who have been trained in OCE techniques may result in consistent early detection of OPC and very likely lower treatment morbidity and mortality from this disease.

\section{Acknowledgements}

Supported in part by grants from the National Institutes of Health, K23 grant (DE016890), Health Future Foundation Faculty Development Grant (\#240046) and The 2013 George F. Haddix President's Faculty Research Fund, Creighton University. The authors thank Ms. Barbara Bittner, Creighton University's Office of Research and Compliance, and Lisa Wee -for help in editing the manuscript.

\section{Funding}

None.

\section{Conflicts of interest}

The authors declare that there is no conflict of interest.

\section{References}

1. U.S. Cancer Statistics Working Group. United States cancer statistics 1999-2010 cancer incidence and mortality data. 2014.

2. Kosary CL, Ries LA, Miller BA, et al. SEER cancer statistics review. 1973-1992 tables and graphs. 1995; pp. 17, 34, 542, 355, 361.

3. Altekruse SF, Kosary CL, Krapcho M, et al. SEER cancer statistics review, 1975-2007. National Cancer Institute. 2010.

4. Murphy GP, Lawrence WJ, Lenhard REJ. Textbook of clinical oncology, $2^{\text {nd }}$ ed. Atlanta, GA: American Cancer Society. 1995

5. Silverman S. Demographics and occurrence of oral and pharyngeal cancers. The outcomes, the trends, the challenge. J Am Dent Assoc. 2001;132(Suppl):7S-11S.

6. Gritz ER, Hoffman A. Behavioral and psycholosocial issues in head and neck cancer. In: Beumer J, et al. editors, Maxillofacial rehabilitation - prosthodontic and surgical considerations. St. Louis: Ishiyaku EuroAmerica. 1996; p. 1-14.

7. Onizawa K, Nishihara K, Yamagata K, et al. Factors associated with diagnostic delay of oral squamous cell carcinoma. Oral Oncol. 2003;39(8):781-788.

8. McLeod NM, Saeed NR, Ali EA. Oral cancer: delays in referral and diagnosis persist. Br Dent J. 2005;198(11):681-684.

9. Moyer VA. Screening for oral cancer: U.S. preventive services task force recommendations statement. Ann Intern Med. 2014;160(1):55-60.

10. Kademani D. Oral cancer. Mayo Clin Proc. 2007;82(7):878-887.

11. Kreppel M, Eich HT, Kubler A, et al. Prognostic value of the sixth edition of the UICC's TNM classification and stage grouping for oral cancer. $J$ Surg Oncol. 2010;102(5):443-449. 
12. Goodman HS, Yellowitz JA, Horowitz AM. Oral cancer prevention. The role of family practitioners. Arch Fam Med. 1995;4(7):628-636.

13. Epstein JB, Gorsky M, Cabay RJ, et al. Screening for and diagnosis of oral premalignant lesions and oropharyngeal squamous cell carcinoma: role of primary care physicians. Can Fam Physician. 2008;54(6):870-875.

14. Patton LL, Agans R, Elter JR, et al. Oral cancer knowledge and examination experiences among North Carolina adults. J Public Health Dent. 2004;64(3):173-180.

15. Allison P, Franco E, Feine J. Predictors of professional diagnostic delays for upper aerodigestive tract carcinoma. Oral Oncol. 1998;34(2):127132

16. Smith RA, Cokkinides V, von Eschenbach AC, et al. American cance society guidelines for the early detection of cancer. CA Cancer J Clin. 2002;52(1):8-22.

17. Brocklehurst P, Kujan O, O Malley LA, et al. Screening programmes for the early detection and prevention of oral cancer. Cochrane Database Syst Rev. 2010;11:CD004150.

18. Rethman MP, Carpenter W, Cohen EE, et al. Evidence-based clinica recommendations regarding screening for oral squamous cell carcinomas. J Am Dent Assoc. 2010;141(5):509-520.

19. Sankaranarayanan R, Mathew B, Jacob BJ, et al. Early findings from a community-based, cluster-randomized, controlled oral cancer screening trial in Kerala, India. The Trivandrum Oral cancer screening study group. Cancer. 2000;88(3):664-673.

20. Parkin DM, Whelan SL, Ferlay J, et al. Cancer incidence in five continents. Lyon: International Agency for Research on Cancer. IARC Scientific Publication no. 143, No. VII. 1997.

21. Subramanian S, Sankaranarayanan R, Bapat B, et al. Cost-effectiveness of oral cancer screening: results from a cluster randomized controlled trial in India. Bull World Health Organ. 2009;87(3):200-206.

22. Poh CF, Hislop G, Currie B, et al. Oral cancer screening in a high-risk underserved community-vancouver downtown eastside. J Health Care Poor Underserved. 2007;18(4):767-778.

23. D Souza G, Kreimer AR, Viscidi R, et al. Case-control study of human papillomavirus and oropharyngeal cancer. $N$ Engl J Med. 2007;356(19):1944-1956.

24. Scully C, Bagan J. Oral squamous cell carcinoma overview. Oral Oncol. 2009;45(4-5):301-308.

25. Kreimer AR, Clifford GM, Boyle P, et al. Human papillomavirus types in head and neck squamous cell carcinomas worldwide: a systematic review. Cancer Epidemiol Biomarkers Prev. 2005;14(2):467-475.

26. Cleveland JL, Junger ML, Saraiya M, et al. The connection between human papillomavirus and oropharyngeal squamous cell carcinomas in the United States: implications for dentistry. $J$ Am Dent Assoc. 2011;142(8):915-924.

27. Brown LM, Check DP, Devesa SS. Oropharyngeal cancer incidence trends: diminishing racial disparities. Cancer Causes Control. 2011;22(5):753-763

28. Speight PM, Palmer S, Moles DR, et al. The cost-effectiveness of screening for oral cancer in primary care. Health Technol Assess. 2006;10(14):1-144.

29. Santana JC, Delgado L, Miranda J, et al. Oral Cancer case finding program (OCCFP). Oral Oncol. 1997;33(1):10-12.

30. Horowitz AM, Nourjah PA. Factors associated with having oral cance examinations among US adults 40 years of age or older. J Public Health Dent. 1996;56(6):331-335.

31. Canto MT, Drury TF, Horowitz AM. Use of skin and oral cancer examinations in the United States, 1998. Prev Med. 2003;37(3):278-282.
32. Horowitz AM, Moon HS, Goodman HS, et al. Maryland adults' knowledge of oral cancer and having oral cancer examinations. J Public Health Dent. 1998;58(4):281-287.

33. MMWR W. Percentage of adults aged $\geq 18$ years who have ever had an oral cancer examination, by smoking status and age group - National Health Interview Survey, United States, 2008. 58(36):1013.

34. CDC/National Center for Chronic Disease Prevention and Health Promotion. Behavioral risk factor surveillance system. Oral health. 2008.

35. Horowitz AM, Drury TF, Goodman HS, et al. Oral pharyngeal cancer prevention and early detection. Dentists' opinions and practices. $J \mathrm{Am}$ Dent Assoc. 2000;131(4):453-462.

36. Gift HC, Newman JF. How older adults use oral health care services: results of a National Health Interview Survey. $J$ Am Dent Assoc. 1993;124(1):89-93.

37. Yellowitz JA, Goodman HS, Farooq NS. Knowledge, opinions, and practices related to oral cancer: Results of three elderly racial groups. Spec Care Dentist. 1997;17(3):100-104.

38. Drilea SK, Reid BC, Li CH, et al. Dental visits among smoking and nonsmoking US adults in 2000. Am J Health Behav. 2005;29(5):462-471.

39. Gornick ME, Eggers PW, Reilly TW, et al. Effects of race and income on mortality and use of services among medicare beneficiaries. $N$ Engl J Med. 1996;335(11):791-799.

40. Reid BC, Warren JL, Rozier G. Comorbidity and early diagnosis of head and neck cancer in a medicare population. Am J Prev Med. 2004;27(5):373-378.

41. Blot WJ, McLaughlin JK, Winn DM, et al. Smoking and drinking in relation to oral and pharyngeal cancer. Cancer Res. 1988;48(11):32823287.

42. Chiodo GT, Eigner T, Rosenstein DI. Oral cancer detection. The importance of routine screening for prolongation of survival. Postgrad Med. 1986;80(2):231-236.

43. Yellowitz JA, Goodman HS. Assessing physicians' and dentists oral cancer knowledge, opinions and practices. J Am Dent Assoc. 1995;126(1):53-60.

44. Kowalski LP, Franco EL, Torloni H, et al. Lateness of diagnosis of oral and oropharyngeal carcinoma: factors related to the tumour, the patient and health professionals. Eur J Cancer Oral Oncol. 1994;30B(3):167173.

45. Canto MT, Horowitz AM, Drury TF, et al. Maryland family physicians knowledge, opinions and practices about oral cancer. Oral Oncol. 2002;38(5):416-424.

46. Applebaum E, Ruhlen TN, Kronenberg FR, et al. Oral cancer knowledge, attitudes and practices: a survey of dentists and primary care physicians in Massachusetts. J Am Dent Assoc. 2009;140(4):461-467.

47. Sohn W, Ismail AI, Kolker JL. Knowledge of oral cancer and screening practices of primary care providers at federally qualified health centers. $J$ Public Health Dent. 2005;65(3):160-165.

48. Elwood JM, Gallagher PR. Factors influencing early diagnosis of cancer of the oral cavity. Can Med Assoc J. 1985;133(7):651-656.

49. Prout MN, Heeren TC, Barber CE, et al. Use of health services before diagnosis of head and neck cancer among Boston residents. Am J Prev Med. 1990;6(2):77-83.

50. Lynch GR, Prout MN. Screening for cancer by residents in an interna medicine program. J Med Educ. 1986;61(5):387-393.

51. Holmes JD, Dierks EJ, Homer LD, et al. Is detection of oral and oropharyngeal squamous cancer by a dental health care provider associated with a lower stage at diagnosis?. J Oral Maxillofac Surg. 2003;61(3):285-291. 
52. de Faria PR, Cardoso SV, de A Nishioka S, et al. Clinical presentation of patients with oral squamous cell carcinoma when first seen by dentists or physicians in a teaching hospital in Brazil. Clin Oral Investig. 2003;7(1):46-51.

53. Yu T, Wood RE, Tenenbaum HC. Delays in diagnosis of head and neck cancers. J Can Dent Assoc. 2008;74(1):61.

54. Siriphant P, Drury TF, Horowitz AM, et al. Oral cancer knowledge and opinions among Maryland nurse practitioners. J Public Health Dent. 2001;61(3):138-144.

55. Meng X, Duncan RP, Porter CK, et al. Florida nurse practitioners' attitudes and practices regarding oral cancer prevention and early detection. $\mathrm{J} \mathrm{Am}$ Acad Nurse Pract. 2007;19(12):668-675.

56. Carter LM, Harris AT, Kavi VP, et al. Oral cancer awareness amongst hospital nursing staff: a pilot study. BMC Oral Health. 2009;9:4.

57. Siriphant P, Horowitz AM, Child WL. Perspectives of Maryland adult and family practice nurse practitioners on oral cancer. J Public Health Dent. 2001;61(3):145-149.

58. LeHew CW, Epstein JB, Koerber A, et al. Training in the primary prevention and early detection of oral cancer: Pilot study of its impact on clinicians' perceptions and intentions. Ear Nose Throat J. 2009;88(1):748-753.
59. Patton LL, Ashe TE, Elter JR, et al. Adequacy of training in oral cancer prevention and screening as self-assessed by physicians, nurse practitioners, and dental health professionals. Oral Surg Oral Med Oral Pathol Oral Radiol Endod. 2006;102(6):758-764.

60. Downer MC, Moles DR, Palmer S, et al. A systematic review of test performance in screening for oral cancer and precancer. Oral Oncol. 2004;40(3):264-273.

61. Scott SE, Rizvi K, Grunfeld EA, et al. Pilot study to estimate the accuracy of mouth self-examination in an at-risk group. Head Neck. 2010;32(10):1393-1401.

62. Elango KJ, Anandkrishnan N, Suresh A, et al. Mouth self-examination to improve oral cancer awareness and early detection in a high-risk population. Oral Oncol. 2011;47(7):620-624.

63. Mehta FS, Gupta PC, Bhonsle RB, et al. Detection of oral cancer using basic health workers in an area of high oral cancer incidence in India. Cancer Detect Prev. 1986;9(3-4):219-225.

64. Mathew B, Sankaranarayanan R, Sunilkumar KB, et al. Reproducibility and validity of oral visual inspection by trained health workers in the detection of oral precancer and cancer. Br J Cancer. 1997;76(3):390-394. 